\title{
Interdependence of Nonoverlapping Cortical Systems in Dual Cognitive Tasks
}

\author{
Marcel Adam J ust,*, ${ }^{1}$ Patricia A. Carpenter,* Timothy A. Keller,* Lisa Emery,* \\ Holly Zajac,* and Keith R. Thulbornt \\ *Center for Cognitive Brain Imaging, Department of Psychology, Carnegie Mellon University, Pittsburgh, \\ Pennsylvania; and HUniversity of Illinois at Chicago, Chicago, Illinois
}

Received August 23, 2000

One of the classic questions about human thinking concerns the limited ability to perform two cognitive tasks concurrently, such as a novice driver's difficulty in simultaneously driving and conversing. Limitations on the concurrent performance of two unrelated tasks challenge the tacitly assumed independence of two brain systems that seemingly have little overlap. The current study used fMRI (functional magnetic resonance imaging) to measure cortical activation during the concurrent performance of two high-level cognitive tasks that involve different sensory modalities and activate largely nonoverlapping areas of sensory and association cortex. One task was auditory sentence comprehension, and the other was the mental rotation of visually depicted 3-D objects. If the neural systems underlying the two tasks functioned independently, then in the dual task the brain activation in the main areas supporting the cognitive processing should be approximately the conjunction of the activation for each of the two tasks performed alone. We found instead that in the dual task, the activation in association areas (primarily temporal and parietal areas of cortex) was substantially less than the sum of the activation when the two tasks were performed alone, suggesting some mutual constraint among association areas. A similar result was obtained for sensory areas as well. 2001 Academic Press

\section{INTRODUCTION}

The recent mapping of cortical areas involved in high level cognitive tasks now permits the direct evaluation of the activation of such areas when two tasks are performed concurrently, as well as when they are performed alone. The ability to monitor and compare the $\mathrm{fMRI}$-measured activity during single and dual task performance may help illuminate the interrelationship

\footnotetext{
${ }^{1}$ To whom correspondence and reprint requests should be addressed. Fax: (412) 268-2804. E-mail: just+ @cmu.edu.
}

between apparently dissociable neural systems. Two systems that are considered somewhat separable based on neuropsychological data (Mesulam, 1990) are the language comprehension system and the visuo-spatial system supporting mental rotation. The neural system supporting sentence comprehension includes the classic language areas, the left superiolateral temporal cortex and left inferior frontal gyrus, and to a lesser extent, homologous areas on the right ( $\mathrm{Just}$ et al ., 1996; Schlosser et al., 1998). By contrast, mental rotation involves the left and right parietal regions and to some extent, the inferior temporal regions (Carpenter et al., 1999; Cohen et al., 1996; Tagaris et al., 1997), areas that are largely nonoverlapping with the classic Ianguage areas. If the functioning of the language and spatial systems were independent, each system would function concurrently in much the same way as they function alone or perhaps with an extra concurrence cost. If they were interdependent, however, then their characteristics when functioning alone should not predict their characteristics when functioning together, as in a dual task.

Several previous neuroimaging studies have examined dual tasks that activate cortical regions that overlap in the two tasks, such as a visually presented verbal task that was performed in the presence or absence of a visual distractor (Rees et al., 1997), or a related task that contrasted attention to single vs multiple visual objects (Vandenberghe et al., 1997). The results of such studies generally show that the activation associated with a given task decreases when a second task, drawing on the same cortical area, is being performed concurrently (Klingberg and Roland, 1997). When a neuroimaging study examined the concurrent performance of two tasks that had different input modalities (a simple spatial relations judgment and a semantic category judgment), the main reported finding was significant dorsolateral prefrontal cortex (DLPFC) activation in the dual but not in the singletask conditions (D’Esposito et al., 1995). 
One feature that distinguishes the current study of dual task performance is its examination of the amount of activation (volume and signal intensity) during the performance of two tasks that entail considerably more complex cognitive computations than the preceding studies. Furthermore, the neural systems engaged by these two tasks are considered dissociable by neuropsychological standards. A third distinguishing feature is that the activation volume in many cortical regions was systematically analyzed.

In the current study, participants performed either a sentence comprehension task alone, a mental rotation task alone, or both tasks simultaneously. To minimize interference at the sensory input level, the sentences were presented auditorily, and the figures to be mentally rotated were presented visually. The comprehension task involved judging general-knowledge sentences, such as The pyramids were burial places and they are one of the seven wonders of the ancient world. F or the rotation task, pairs of drawings of abstract 3-D figures had to be mentally rotated to judge their identity (Shepard and Metzler, 1971). The presumption that these two tasks draw on nonoverlapping areas of association cortex is verified in the current study. There was very little activation in the classic language areas during the rotation task, and little activation in the parietal and ventral temporal regions during the language comprehension task. The critical question concerned the concurrent performance of the tasks. The prediction from the independence assumption is that the amount of fMRI-measured activation in the critical association cortex regions during the concurrent performance of the two tasks should equal or slightly exceed the amount when each task is performed in isolation. By contrast, if the underlying language and spatial systems were inter-dependent, despite their lack of anatomical overlap, then their functioning in a dual task should be something other than the sum of their separate functioning. In particular, if they both depend on a common resource pool, then the activation volume in the dual task condition should be less than the sum of the activation volume in the two single-task conditions.

\section{MATERIALS AND METHODS}

\section{Task and Stimulus Materials}

In the rotation task, participants performed a mental rotation task involving drawings of complex, 3-D figures originally used by Shepard and Metzler (1971). In the current study, the two figures were the same (with disparities of either 40 or $80^{\circ}$ ) on two-thirds of the trials and different (mirror-image isomorphs) on the other third. Participants signaled their binary response using two handheld pushbuttons operated by their left thumb. Failure to respond within $5.5 \mathrm{~s}$ was

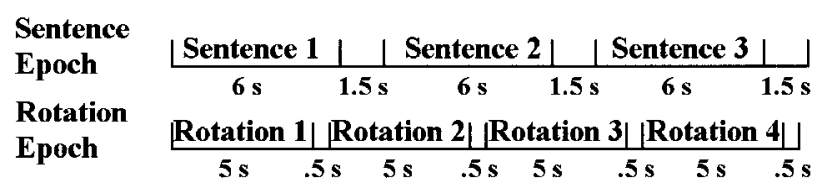

FIG. 1. A schematic diagram of the timing of a dual task epoch involving four items of the mental rotation task and three sentences in the comprehension task. In the rotation task, each pair of figures was presented for $5.0 \mathrm{~s}$, with a 0.5 -s pause between items. In the comprehension task, each auditory sentence presentation was about $6 \mathrm{~s}$, with a 1.5-s interval between items.

treated as an error. The figures were presented in a sequence of four items each, lasting $22 \mathrm{~s}$ (an epoch).

In the sentence comprehension task, participants listened to general knowledge sentences that they verified as true or false. E ach sentence took approximately $6 \mathrm{~s}$ to articulate, and a single sentence epoch contained three sentences and lasted $22.5 \mathrm{~s}$. A short tone sounded at the end of each sentence, and failure to respond within the next $3 \mathrm{~s}$ was treated as an error. Two-thirds of the sentences were true and one-third were false, and participants signaled their response using two handheld pushbuttons operated by their right thumb. The sentences were stored as digitized files and were presented using pneumatic transmission through plastic tubing terminated with earphones.

The timing of the mental rotation task and sentence comprehension task is depicted in Fig. 1. In the dual task epochs, the rotation stimuli and sentences started simultaneously and had the same timing as in the single tasks. Because there were four rotations and three sentences per epoch, the responses to the two types of items generally did not occur at the same time.

The experiment consisted of 8 epochs of each of the single tasks (sentence comprehension and mental rotation) and 16 epochs of the dual task. Participants were asked to perform both tasks equally conscientiously in the dual condition. The order of the 32 experimental epochs was balanced to minimize differential order effects; the quartiles of the stimulus presentations were in the order: single, dual, single, dual, so that order effects could be assessed. A 6-s rest occurred between each epoch. In addition, after every four epochs, there was a 24-s fixation condition in which the participant fixated a centered asterisk without performing any task; the fixation condition constituted a baseline measure of brain activation with which to compare experimental conditions.

\section{Subjects}

Eighteen right-handed native English speakers (6 females), ages 18 to 32, who showed less than $40 \%$ error in each condition (namely $60 \%$ of all screened individuals), participated after signing a written consent form that had been approved by University of Pittsburgh and Carnegie Mellon Institutional Review 


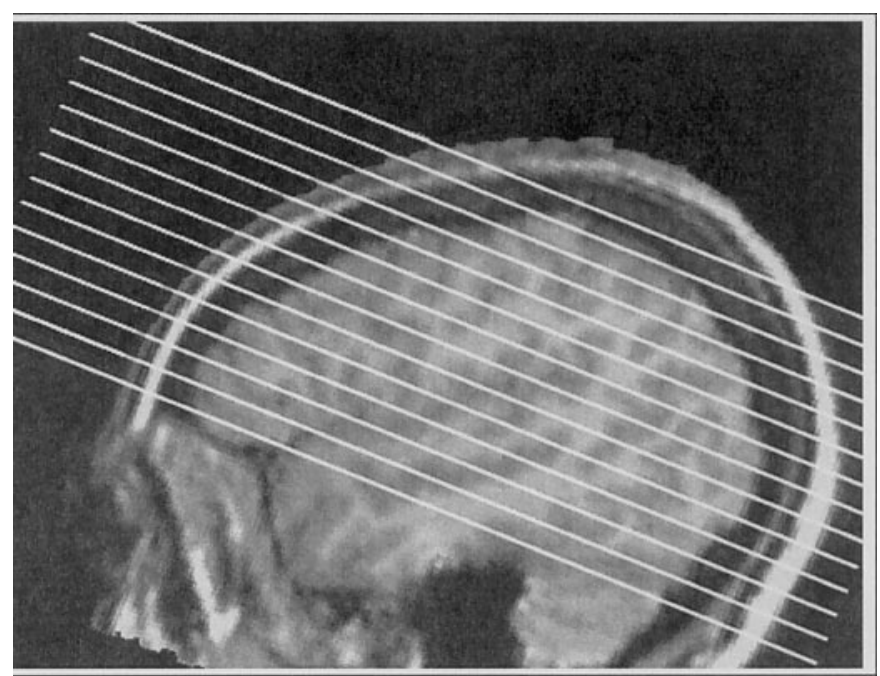

FIG. 2. Cortical coverage of 14 oblique-axial slice prescription for a typical participant shown in a very lateral sagittal view. In more medial areas, much more of the cerebellum is covered.

Boards. Data from 10 other participants were discarded because of excessive head motion or technical problems, and from 1 because of inaccurate task performance.

\section{Scanning Procedures}

The gradient echo, resonant echo planar fMRI used BOLD (blood oxygen level-dependent) contrast in a 3.0 Tesla GE Medical Systems scanner at the MR Research Center of the University of Pittsburgh Medical Center. I mages were acquired in 14 adjacent oblique axial planes (with a pitch angle ranging from 9 to $21^{\circ}$ to maximally cover the parietal and temporal lobes) (see Fig. 2) every $3000 \mathrm{~ms}$ (TR or repetition time), with $\mathrm{TE}=25 \mathrm{~ms}$, flip angle $=90^{\circ}$, and voxel sizes of $3.125 \times$ $3.125 \times 5 \mathrm{~mm}$, and using a GEMS quadrature birdcage head coil. The field of view was $400 \times 200 \mathrm{~mm}$, a $128 \times$ 64 acquisition matrix, 5-mm slice thickness, 1-mm gap.

\section{Data Analysis}

I mage preprocessing (including baseline correction, deghosting, mean correction, motion correction, and trend correction) was performed using FIASCO (Eddy et al., 1996; Lazar et al., 2001) (further description and tools are available at www.stat.cmu.edu/ fiasco/). The mean of the maximum head motion per participant was 0.3 voxels, and it never exceeded 0.6 voxels. To accommodate the rise and fall time of the hemodynamic response (Bandettini et al., 1992), data from the first $6 \mathrm{~s}$ of each epoch and the 6 -s rest interval between epochs were discarded. A voxel was considered activated in a condition if a t test comparing its activation in that condition to its level during the fixation condition reached a threshold value of $t>6.0$, which is more conservative than the Bonferroni correction for $\mathrm{P}<$ 0.01 . Excluded from consideration were the $1 \%$ of all voxels showing more than a $6 \%$ change in signal intensity that might have possibly arisen from blood vessels. ${ }^{2}$

Regions of interest (ROIs) were defined anatomically a priori for each participant, using a sulcusoriented parcellation scheme and nomenclature (Rademacher et al., 1992). The ROI s were defined with reference to coregistered structural images, high resoIution T1-weighted 3-D SPGR volume scans with TR = $25 \mathrm{~ms}, \mathrm{TE}=4 \mathrm{~ms}, 256 \times 256$ acquisition matrix, 1.5-mm slice thickness, no gap. One analysis focused on the four regions where most of the association area activation occurred: left and right parietal and temporal regions. The parietal ROIs included the superior parietal lobule, the anterior and posterior supramarginal gyrus, and the angular gyrus (areas SPL, SGa, $\mathrm{SG}$, and AG). The temporal ROIs included both the superior and middle temporal gyri (T1a, T1p, T2a, T2p, TO2). The anatomical information in the structural images was displayed in the three orthogonal planes simultaneously and the ROIs were manually drawn on each functional slice. The interrater reliability of this ROI-defining procedure between two trained staff members was evaluated for four ROI's in two participants in another study. The reliability measure was obtained by dividing the size of the set of voxels that overlapped between the two raters by the mean of their two set sizes. The resulting eight reliability measures were in the $78-91 \%$ range, with a mean of $84 \%$, as high as the reliability reported by the developers of the parcellation scheme.

A second analysis focused on five sensory areas: the primary auditory ROI was Heschl's gyrus ( $\mathrm{H} 1)$; the primary visual ROI included the calcarine cortex, extending to the occipital pole (CALC, SCAL, OP), and the secondary visual ROI included all of occipital cortex that was not in the primary visual ROI, plus the posterior portions of inferior temporal gyrus (T3p, TO3, OLi, OLs, TOF, OF, OP, LG).

The third analysis focused on eight prefrontal regions. The dorsolateral prefrontal cortex (DLPFC) ROI was defined as the portion of the middle frontal gyrus (F2) that was anterior to and excluded the precentral sulcus. The frontal eye fields (FEF) included the portion of the precentral sulcus posterior to the middle frontal gyrus (F2), as well as the two posterior-most voxels of $\mathrm{F} 2$ itself. The anterior cingulate gyrus ROI was CGa. The inferior frontal gyrus ROI included pars

\footnotetext{
${ }^{2}$ To assure that the elimination of those voxels that had a signal change greater than $6 \%$ did not bias the results against one of the conditions, the analyses reported below for all ROI's were repeated with all voxels included, and the results were very similar to those reported; all reliable differences remained reliable and no additional differences became reliable.
} 


\section{Language Task Alone \\ Language in Dual Task}
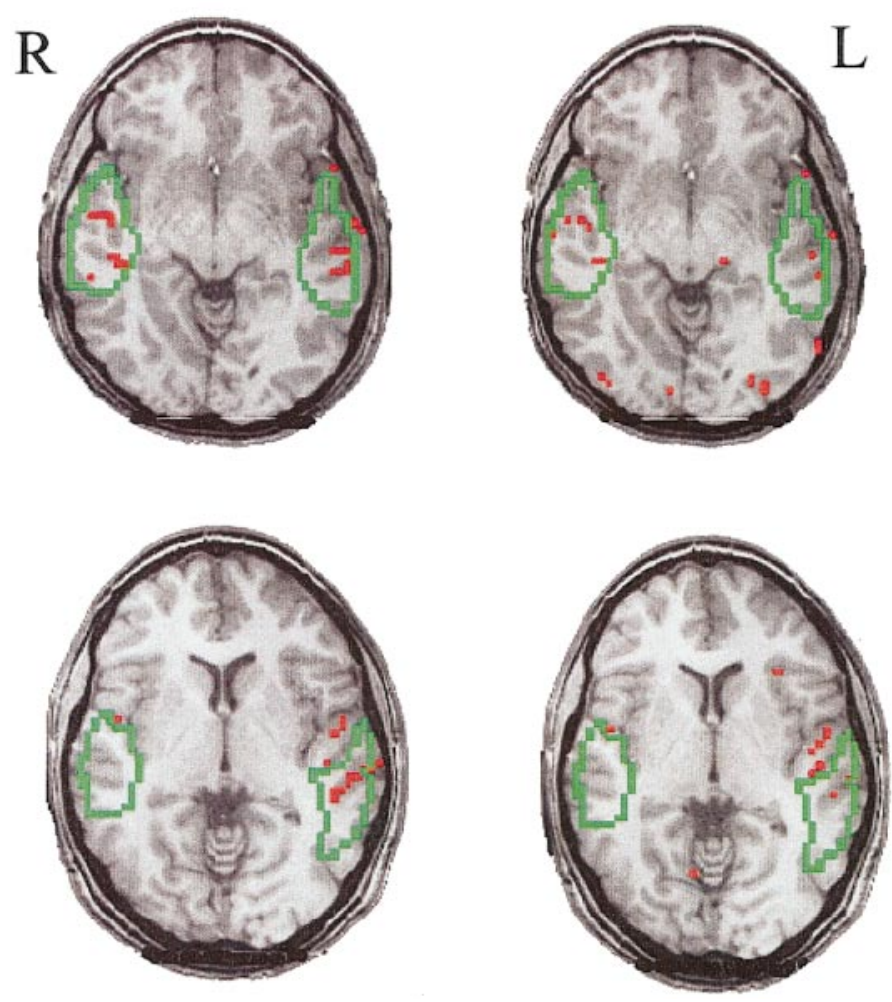

FIG. 3. Thresholded fMRI brain activation images (superimposed on structural images) comparing a single task comprehension condition (left-hand column) to a dual task condition, for the slice showing most temporal activation, in two participants. The number of activated voxels in the superiolateral temporal cortex ROIs (green border) decreases from the single task condition to the dual task condition.

opercularis and pars triangularis (F 3t and F 30). The paracingulate/medial frontal ROI included the paracingulate gyrus, the inferior portion of $\mathrm{F} 1$ and was extended to the frontal pole (PAC, F 1, FP). Besides these three foci of analysis, four additional ROI's were defined to capture the remaining activation: the motor ROIs consisted of the precentral gyrus including the central sulcus (PRG and ce), and the supplementary motor ROI consisted of the J uxtaparacentral Lobule Cortex (J PL). The cerebellar ROI consisted of all of that structure captured by the slice prescription.

\section{RESULTS}

The behavioral measures indicated that the dual tasks were performed without compromising accuracy in either task. F or example, one way to deal with a dual task is to simply ignore one of the tasks and choose responses randomly, but then the response accuracy would be at chance level (50\% here). Contrary to any such approach, the response accuracies in the dual task were $85.9 \%$ for the sentence task and $87.7 \%$ for the rotation task, indicating that both tasks were being performed with a high degree of conscientiousness. At the same time, the performance was not quite as good in the dual as in the single task conditions. For sentence comprehension, the performance declines were negligible from single to dual task in terms of error rates (14.4\% and $14.1 \%)$, but there was a reliable increase in the response times measured from the end of the sentence (532 and $740 \mathrm{~ms}), F(1,17)=24.8, P<$ 0.01 . For mental rotation, the performance declines were reliable from single to dual task in terms of both error rates $(7.8$ and $12.3 \%, F(1,17)=10.69, \mathrm{P}<0.01)$ and total response times $(2440$ and $2792 \mathrm{~ms},(F(1,17)=$ 23.72, $\mathrm{P}<0.01$ ).

The main $\mathrm{fMRI}$ finding was that the amount of activation in the most involved areas of association cortex, namely temporal and parietal lobe areas, was substantially less in the dual task than in the sum of the two single tasks. The two tasks performed together pro-

\section{Rotation \\ Task Alone}
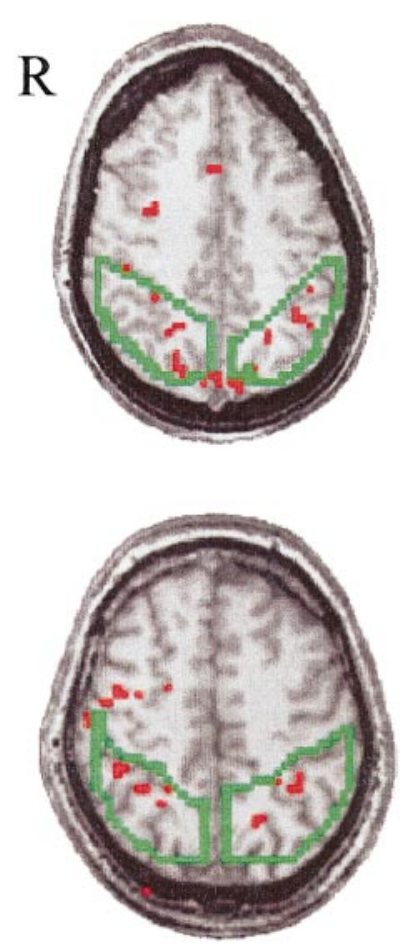

Rotation in Dual Task
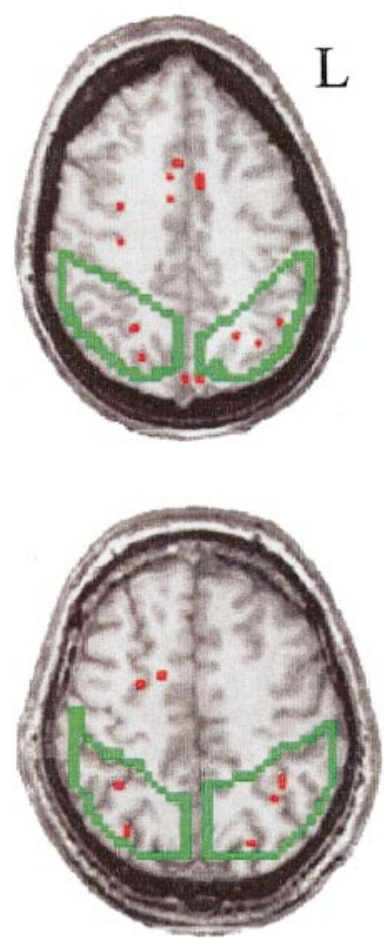

FIG. 4. Thresholded $f M R I$ brain activation images (superimposed on structural images) comparing the single task mental rotation condition (left-hand column) to the dual task condition, for the slice showing most parietal activation, in two participants. The number of activated voxels in the parietal ROIs decreases from the single task condition to the dual task condition. 
duced only $56 \%$ as much activation volume in the temporal and parietal lobes as the sum of the two single task conditions (41.6 voxels vs 74.5 voxels), $\mathrm{F}(1,17)=$ 24.0, $\mathrm{P}<0.01$. This difference occurred in 16 (of 18) participants, with almost no difference in a 17th participant. Figures 3 and 4 show representative decrements in activation in both tasks in a single slice through the relevant regions for two participants. Figure 5 shows how the mean activation volume in these regions (averaged across participants and measured in terms of the number of activated voxels) decreases from the single to dual-task conditions. The decrement in activation was larger in the language-related cortical areas (left and right superior and middle temporal regions, excluding the auditory sensory area of Heschl's gyrus); the mean number of activated voxels here decreased reliably from 34.1 in the language-comprehension only condition to 16.6 in the dual conditions, $\mathrm{F}(1,17)=43.17, \mathrm{P}<0.01$. The decrement was smaller but still substantial in the spatial processing areas (left and right parietal), where the mean number of activated voxels decreased reliably from 35.1 to 25.0 voxels $(\mathrm{F}(1,17)=4.49, \mathrm{P}<0.05)$. Table 1 shows the amount of activation in each $\mathrm{ROI}$ in each condition, as well as the average centroids of activation in the dual task condition, which were similar to the single task conditions. ${ }^{3}$

The decrement in activation from the single to dual task conditions is particularly notable in light of the relative non-overlap of activation between the two sin-

\footnotetext{
${ }^{3}$ To assure that the main findings were not due to the order of presentation of the single and dual tasks (such that the dual tasks could have differentially benefited from occurring in the second and fourth quartile of the study), we compared the results from the first occurrence of the dual task (second quartile) with the second occurrence of the single tasks (third quartile), and the main results still held. To determine the dependence of the main finding on the particular choice of threshold of $t>6$, the comparisons of the activation volume in the dual task versus the sum of the single tasks were repeated using a range of thresholds from $t>5$ to $t>7$. Although the absolute number of voxels activated depended considerably on the choice of threshold, producing a threefold decrease in the number of voxels activated from $t>5$ to $t>7$, the relative amounts of activation in the single and dual task conditions remained fairly stable. Whereas at a threshold of $\mathrm{t}>6$ the two tasks performed together produced $56 \%$ as much activation volume in the temporal and parietal lobes as the sum of the two single task conditions, as reported above, the percentages at the thresholds of $t>5$ to $t>7$ were $66 \%$ and $48 \%$ respectively. (The results for $t>5$ probably contain too many false positives, but the relation between dual and single tasks still holds). At all three thresholds, the results are considerably and reliably different from the null hypothesis prediction of $100 \%$ as much activation in the dual task as in the sum of the single tasks. Thus the result of less activation in the dual task is not thresholddependent. Although there are comparisons for 21 ROI's being re ported in Table 1, we believe that applying a Bonferroni correction throughout would be too conservative, particularly in view of the a priori hypotheses concerning the two temporal and two parietal ROI's. Were a Bonferroni correction to be applied in the remaining ROI's, which we still view as excessively conservative, then several of the comparisons would no longer be reliable, namely, in left and right Heschl's gyrus, and left secondary visual.
}

هParietal $\square$ Temporal

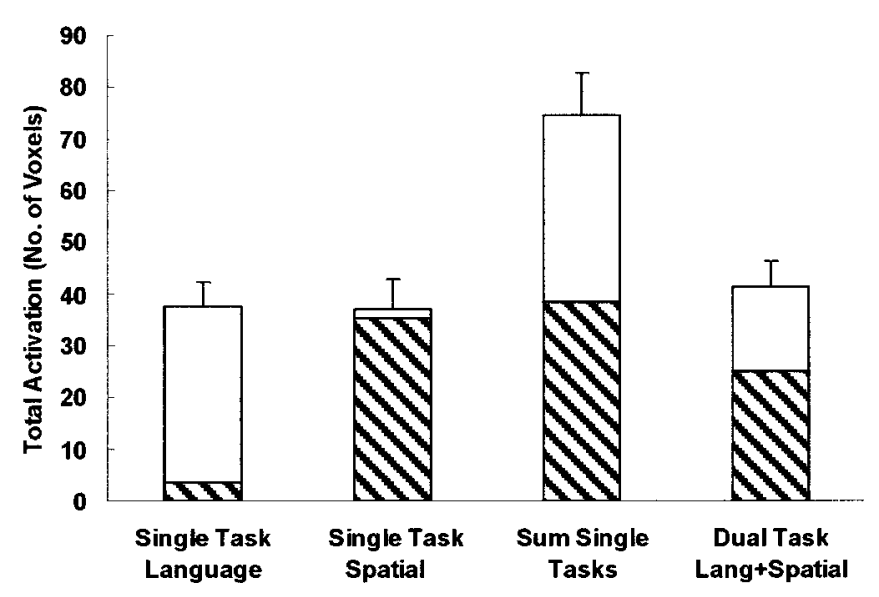

FIG. 5. The mean number of voxels activated in each of the two single-task conditions, the sum of the two single-task conditions, and in the dual task condition, for the language areas (left and right superior/middle temporal regions, open bars) and in the spatial processing areas (left and right parietal lobules, striped bars) (and standard errors of the means over 18 participants). The number of activated voxels in the dual task is substantially less than the sum of the two single task conditions.

gle tasks. The language task alone activated very little of the parietal areas (3.5 voxels, mostly in the angular gyrus) compared to 35.1 voxels in the rotation-only condition (primarily around the intraparietal sulcus); the rotation task alone activated very little of the superior and middle temporal areas (1.8 voxels compared to 34.1 voxels in the language only condition).

The activation was fairly symmetric between the left and right hemispheres, with only slightly more left than right posterior temporal activation (17.6 vs 16.5 voxels) for the language task and conversely, slightly more right than left parietal activation (19.1 vs 16.0 voxels) for the spatial task. Note that auditory sentence comprehension is considerably less left-lateralized than visual sentence comprehension when compared in a within-subjects design (Michael et al., in press).

Sensory areas. The underadditivity of the dual task activation (relative to the single task activation) also occurred in the primary and secondary sensory areas. The sensory areas for sentences included Heschl's gyrus, and for rotation items, primary visual cortex (calcarine sulcus to the occipital pole), and secondary visual areas. The activation in all these sensory areas was substantially less in the dual task than in the sum of the two single tasks. The two tasks performed together produced only $62 \%$ as much activation volume as the sum of the two single task conditions (44.0 voxels vs 70.6 voxels), a highly reliable difference $(F(1,17)=17.81, P<0.01)$, as shown in Fig. 6. (Note that the rotation task alone produced no activation in 
TABLE 1

Activation Volume in Single and Dual Tasks

\begin{tabular}{|c|c|c|c|c|c|c|c|c|}
\hline \multirow[b]{2}{*}{ ROI } & \multirow{2}{*}{$\begin{array}{l}\text { Sentence } \\
\text { task }\end{array}$} & \multirow{2}{*}{$\begin{array}{l}\text { Rotation } \\
\text { task }\end{array}$} & \multirow{2}{*}{$\begin{array}{l}\text { Sum single } \\
\text { tasks }\end{array}$} & \multirow{2}{*}{$\begin{array}{l}\text { Dual } \\
\text { task }\end{array}$} & \multirow[b]{2}{*}{$t^{a}$} & \multicolumn{3}{|c|}{ Talairach coordinates $^{b}$} \\
\hline & & & & & & $x$ & $y$ & z \\
\hline \multicolumn{9}{|l|}{ Parietal/temporal } \\
\hline L Temporal & 17.6 & 0.7 & 18.3 & 7.9 & $6.49 * *$ & -52 & -19 & 6 \\
\hline R Temporal & 16.5 & 1.1 & 17.6 & 8.7 & $5.51^{* *}$ & 51 & -21 & 5 \\
\hline L Parietal & 2.0 & 16.0 & 18.0 & 11.8 & $2.24^{*}$ & -29 & -52 & 44 \\
\hline $\begin{array}{l}\text { R Parietal } \\
\text { Sum }\end{array}$ & 1.5 & 19.1 & 20.6 & 13.2 & $3.06 * *$ & 29 & -54 & 42 \\
\hline Pariet./Temp. & 37.6 & 36.9 & 74.5 & 41.6 & $4.90 * *$ & & & \\
\hline \multicolumn{9}{|l|}{ Sensory } \\
\hline L Heschl's & 4.8 & 0.0 & 4.8 & 2.9 & $2.55^{*}$ & -49 & -16 & 11 \\
\hline R Heschl's & 2.6 & 0.0 & 2.6 & 1.4 & $2.15^{*}$ & 49 & -14 & 12 \\
\hline Primary Visual & 1.3 & 15.7 & 17.0 & 10.2 & $3.80 * *$ & 3 & -72 & 8 \\
\hline L Sec. Visual & 1.6 & 20.7 & 22.3 & 14.2 & $2.84^{*}$ & -26 & -70 & 3 \\
\hline R Sec. Visual & 0.8 & 23.1 & 23.9 & 15.3 & $3.80 * *$ & 32 & -67 & 4 \\
\hline Sum Sensory & 11.1 & 59.5 & 70.6 & 44.0 & $4.22 * *$ & & & \\
\hline \multicolumn{9}{|l|}{ Prefrontal } \\
\hline L DLPFC & 0.9 & 0.5 & 1.4 & 1.6 & n.s. & -34 & 25 & 35 \\
\hline R DLPFC & 0.4 & 1.4 & 1.8 & 1.7 & n.s. & 32 & 33 & 35 \\
\hline Ant. Cing. & 0.7 & 0.6 & 1.3 & 1.9 & n.s. & 0 & 13 & 35 \\
\hline L Inf. Fr. Gyrus & 1.3 & 0.8 & 2.1 & 1.6 & n.s. & -37 & 18 & 18 \\
\hline R Inf. Fr. Gyrus & 1.1 & 1.6 & 2.7 & 2.0 & n.s. & 40 & 21 & 13 \\
\hline L FEF & 1.4 & 1.1 & 2.5 & 2.5 & n.s. & -40 & -1 & 45 \\
\hline R FEF & 0.4 & 1.4 & 1.8 & 1.8 & n.s. & 38 & 2 & 41 \\
\hline Paracing. & 2.5 & 1.6 & 4.1 & 3.7 & n.s. & -1 & 16 & 48 \\
\hline Sum Prefrontal & 8.7 & 9.0 & 17.7 & 16.8 & n.s. & & & \\
\hline \multicolumn{9}{|l|}{ Motor } \\
\hline L Motor & 1.6 & 2.4 & 4.0 & 3.0 & n.s. & -35 & -8 & 49 \\
\hline R Motor & 0.6 & 3.9 & 4.5 & 3.0 & $2.92 * *$ & 37 & -5 & 45 \\
\hline Supp. Motor Area & 0.3 & 0.4 & 0.7 & 1.1 & n.s. & 1 & -2 & 56 \\
\hline Sum Motor & 2.5 & 6.7 & 9.2 & 7.1 & $2.13^{*}$ & & & \\
\hline \multicolumn{9}{|l|}{ Cerebellum } \\
\hline Cerebellum & 0.7 & 6.0 & 6.7 & 10.7 & n.s. & 1 & -55 & -12 \\
\hline
\end{tabular}

${ }^{a} \mathrm{t}$ statistic comparing dual task to sum of single tasks.

${ }^{\mathrm{b}}$ Mean of subjects' centroids in dual task; positive numbers indicate right ( $\mathrm{x}$ ), anterior (y), and superior ( $\mathrm{z}$ ) directions.

$* \mathrm{P}<0.05 ; * * \mathrm{P}<0.01$.

Heschl's gyrus, and the auditory sentence task alone produced only a minimal amount of activation in the primary and secondary visual areas, perhaps due to encoding of a visual fixation point.)

Prefrontal areas. The pattern of activation in the prefrontal areas differed from that in the other areas in three ways. First, the amount of activation was very small, an average of about 1 activated voxel in each of 8 frontal ROIs in the single tasks. Second, this was an area of overlap, such that both single tasks produced activation here. And third, the activation was additive; across the 8 ROI s in the single tasks there were 8.7 activated voxels for the sentence task and 9 voxels for the rotation task, which when summed is similar to the 16.8 voxels observed in the dual task, as shown in Fig. 7.

The prefrontal area that would most be expected to show substantial activation in the dual task is the dorsolateral prefrontal cortex (DLPFC) (D'Esposito et al., 1995). However, the amount of activation here was minimal in both single and dual-task conditions. The number of activated voxels for the sum of the two single tasks (1.8 and 1.4 for right and left DLPFC, respectively) was similar to that for the dual task (1.7 and 1.6 voxels, respectively).

The most activated prefrontal area, which still had only a very small amount, was the paracingulate/medial superior frontal area, with 4.1 voxels in the sum of the single tasks and 3.7 voxels in the dual task. The two frontal eye fields similarly showed a small amount of activation, with 4.3 voxels (left and right combined) for the sum of the single tasks and the same amount (4.3 voxels) for the dual task. Activation in the inferior frontal gyri was also small in the sum of the single tasks (4.8 voxels, left and right combined) and in the dual task (3.6 voxels). Anterior cingulate activation was small for the sum of the single tasks (1.3 voxels) and in the dual task (1.9 voxels). Like the prefrontal regions, the primary and supplementary motor regions showed approximate additivity, with 9.2 voxels for the 
DHeschl's Gyrus $\square$ Primary Visual $\square$ Secondary Visual

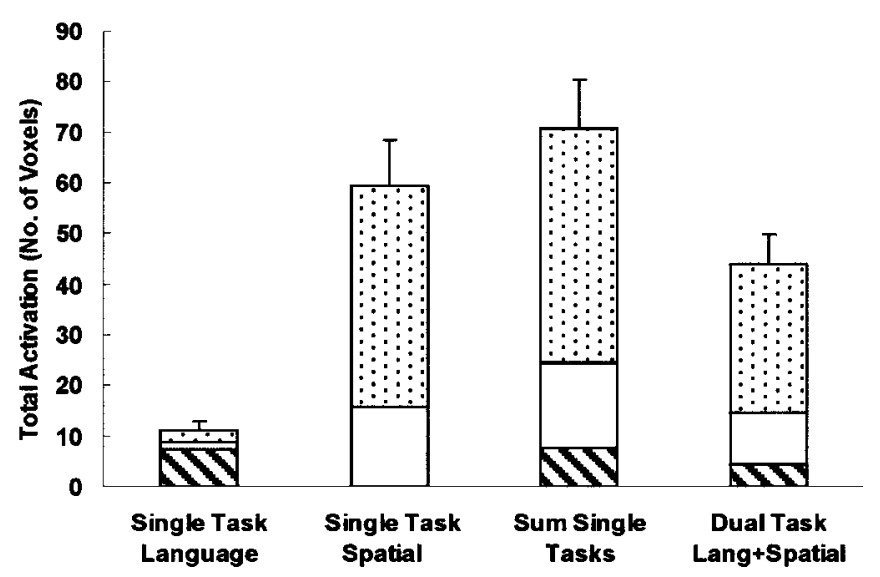

FIG. 6. The mean number of voxels activated in each of the two single-task conditions, the sum of the two single-task conditions, and in the dual task condition, for the primary auditory sensory area (Heschl's gyrus, striped bars), for the primary visual area (open bars) and the secondary visual areas (stippled bars) (and standard errors of the means over 18 participants). The number of activated voxels in the dual task is substantially less than the sum of the two single task conditions.

sum of the single tasks, and 7.1 voxels for the dual task.

The cerebellum was one of the few areas to show even a hint, al beit still statistically unreliable, of more activation in the dual task (10.7 voxels) than in the sum of the single task conditions (6.7 voxels). Its coverage was often incomplete in the slice prescription, and of course, it was the only region outside of the cortex.

Importantly, the ROIs collectively include most of the activated voxels in the entire volume defined by all 14 of the 5-mm oblique axial slices that covered most of the cortex. Consequently, it is possible to compare the total sum of activation aggregated across ROIs for the two single tasks and the dual task. Such a comparison shows the same underadditive pattern as we have shown for the association and sensory areas. The total amount of activation in the entire imaged volume from the single sentence task was on average 68 voxels; the total activation was greater for the single rotation task (128.2 voxels) because the activation in the visual sensory regions was much greater than in the auditory sensory regions. The total activation in the dual task (137.2 voxels) was similar to that for the rotation task alone, indicating only a small increment of activation volume in performing two tasks as compared to one.

The reports above describe how performing two tasks concurrently results in a much smaller number of voxels (41.6) in the temporal and parietal ROIs reaching a fixed (high) threshold, compared to performing the single tasks (74.5 voxels). One can also ask how performing a dual task affects the intensity of the activation of the participating voxels. Is the level of the activation lower in the dual task than in the single tasks? In the temporal and parietal areas, the percentage change in signal intensity (relative to the baseline fixation condition) was reliably lower in the dual task, as follows. In the temporal ROIs the intensities were 3.07 and 2.93 in the language and dual tasks, respectively, a small but reliable difference $(F(1,17)=4.57$, $\mathrm{P}<0.05)$. Similarly, in the parietal ROls, the intensities were 3.07 and 2.86 in the rotation and dual tasks, respectively, another small but reliable difference $(\mathrm{F}(1,17)=9.95, \mathrm{P}<0.01)$. Thus dual task performance considerably reduces the number of highly activated voxels in these areas, while at the same time producing a modest decrease in the activation level of those voxels that are activated in each condition. In the sensory areas, which all showed a reliable decrease in the number of activated voxels, there were no reliable decreases in signal intensity from the single to the dual task conditions, except for the secondary visual areas (which showed a decrease from 3.41 to $3.23 \%, F(1,17)=$ $4.63, \mathrm{P}<0.05)$. The comparisons of intensities in the prefrontal areas could not be made because many participants failed to show reliable activation in those areas. In summary, the signal intensity analyses indicate that whatever constraint on activation is being imposed in the dual task conditions is manifested primarily in the activation volume and less so in the activation intensity of the activated areas.

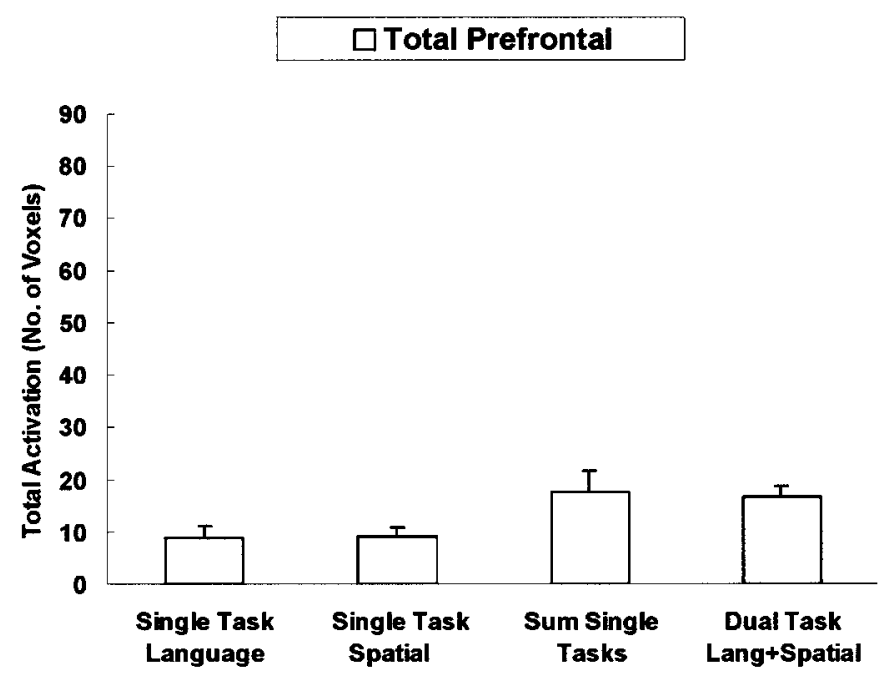

FIG. 7. The mean number of voxels activated in each of the two single-task conditions, the sum of the two single-task conditions, and the number in the dual task condition, for all of the prefrontal areas combined (and standard errors of the means over 18 participants). Although the number of activated voxels is relatively low overall, the number of activated voxels in the dual task is similar to the sum of the two single task conditions. 


\section{DISCUSSION}

When mental rotation and sentence comprehension are performed concurrently, the sum of the activation volume in the major association areas is significantly less than the sum when each task is performed alone. These results are particularly striking in view of the relative lack of activation of the parietal lobes by the language task alone and the lack of superior/middle temporal activation by the rotation task. The relative dissociation between the tasks when performed alone at first suggests a relative independence of function between the two regions. Nevertheless, when the tasks are performed concurrently, the activation volume in the cortical systems underlying the two tasks is not independent, but decreases relative to the single task conditions.

The interpretation that we offer for this finding entails several different perspectives of what may be a single underlying phenomenon. The interpretations pertain to constraints on brain activation, on attention, and on performance. The interpretation that is closest to the results is that there may be a constraint on the total amount of brain activation in association areas and sensory areas. Another way to state this is that there may be biological mechanisms that place an upperbound on the amount of cortical tissue that can be activated at any given time. What such mechanisms might be is not clear, but candidate mechanisms might include metabolic processes, or some neurotransmitter or neuromodulator function. Also consistent with the notion of a biological constraint are electrophysiological measures of brain activity (event-related scalp potentials), which show a decrease in amplitude when an "independent" secondary task is added to a primary task (Wickens et al., 1983). The electrophysiological findings provide converging evidence from another type of measure of less brain activity in a dual task. The possibility of a biological constraint raises the question of whether there might be a single constraint that applies to both association and sensory areas, or a separate constraint that applies to each type of area. There may be separate constraints on activation for various brain systems, systems which could be defined in terms of anatomy, physiology, or functional connectivity or some other type of partition basis. Alternatively, there may be a single, cortex-wide or brain-wide constraint on thetotal amount of activation that can be supported at any given time or perhaps over some time interval, as suggested by the similarity between the total activation volume in the rotation task and the dual task.

Another interpretation that may be closely related to the one above is that there is a limit on how much attention is available to distribute over more than one task. The word attention is used in several senses, one of which refers to a limited cognitive commodity that can be distributed over tasks, such as in divided attention tasks. The current study provides a bridge to functional theories of this sense of attention, providing an explanation at another level of analysis of the possible source or nature of the attention limitation, suggesting why one can't attend to and perform many tasks simultaneously. ${ }^{4}$ The cognitive or attention limitation in the dual task is accompanied by a brain activation decline. It is reasonable to speculate that they are both manifestations of the same phenomenon. The limitation on the cognitive resource referred to as "attention" may be a manifestation of a limit on brain activation.

The third related perspective is that there is a limit on how well it is possible to perform concurrent tasks. The behavioral results showed that although both concurrent tasks were performed at a high absolute level of accuracy, the behavioral performance was reliably poorer in the dual task conditions. This phenomenon may be related to the two perspectives above. The lower amount of activation and the lower amount of attention per task in the dual task condition could be the "cause" of the somewhat poorer performance in the dual task condition. Thus all three perspectives may be different facets or levels of explanation of the same phenomenon. The brain activation perspective brings a new insight to these constraints, namely by demonstrating interarea or intersystem constraints.

The findings raise a number of new and interesting questions. For example, one interesting issue raised by the brain imaging results concerns the possibility that the distribution of brain activation between two tasks may be amenable to strategic control, as it is with respect to the control of other aspects of attention, such as attention to visual locations (Corbetta, 1998). To determine whether the allocation of brain activation to two tasks could be modulated by instructions, an ancillary study (in preparation) presented both tasks simultaneously with instructions to divide attention in various ways between these two tasks. The results of that study demonstrated that the activation volume was distributed in a way that corresponded to the attention-dividing instructions.

Another interesting question posed by the results concerns how it was possible for participants to perform the tasks in the dual condition at high levels of accuracy despite the much lower volumes of activation (compared to the single task conditions). The reduced activation in the dual task may reflect the use of a

\footnotetext{
${ }^{4}$ One type of limitation that the current study attempted to minimize is concerned with competition for the same neural or physical system for processing the sensory input and the motor output associated with the two tasks. Different sensory modalities and different limbs for responding were used in the two tasks. Competition for the same input or output mechanism at either the neural or the physical level is a competition for a somewhat different kind of limited re source.
} 
sampling strategy that would enable the participants to encode and process less information per unit time, while performing at roughly comparable levels of accuracy and speed in the single and dual task conditions. For example, the auditory sentence comprehension task is probably data-limited (Norman and Bobrow, 1975), allowing a participant some time between the end of the processing of each successive word and the onset of the next word. In the dual-task condition, a participant may have sampled and processed the auditory input less frequently (thus, generating less brain activation) than in the single-task condition and still have been able to accurately judge the sentence's truth value. If either task had imposed more computational demand per unit time (say, by presenting the sentence at a faster rate or requiring a larger angular rotation in the same time interval), then a deterioration in accuracy would probably have been more noticeable in the dual task. In other words, the constraint on co-processing may apply not to the number of tasks that can be performed simultaneously, but to the amount of computation performed per unit time in each task. This new interpretation helps to explain why it is increasingly possible to concurrently perform multiple tasks, such as driving and conversing, as one or both become automated and less resource demanding. However, even for an experienced driver, a sudden, computation-demanding complexity in traffic events can put an end to conversation, and a complex conversation may put an end to careful driving.

The results here cast a somewhat new light on the role of prefrontal cortex in dual tasks. A previous study (D'E sposito et al., 1995), unlike the current one, found DLPFC to be very substantially activated during the concurrent performance of two simple tasks but not in either single task alone. The two tasks in that study were a semantic category judgment involving single words and a judgment of the spatial relations in a simple 2-D pattern. These tasks are simpler than the current ones and performed more quickly ( 1 word/2 s for a semantic judgment, 1 picture/3 s for a spatial judgment). As the authors of that study noted, the degree of activation in DLPFC in that dual task situation may reflect a coordinating role in rapidly switching between the items of the two tasks. By contrast, in the current dual task, the items overlapped over much longer time intervals, making discrete switching less likely and perhaps obviating the need for substantial DLPFC participation. Furthermore the dual-task DLPFC activation in D'Esposito et al.'s study was interpreted as potentially reflecting the involvement of the central executive system proposed by Baddeley's theory (1986) of working memory. Another possible role of DLPFC in dual task performance could be related to response inhibition. However, the current findings show excellent concurrent performance of two complex tasks with little additional DLPFC involve- ment. In other words, the performance of two concurrent tasks can occur without involving significant activation of a substantial third area or system. Whatever prefrontal-supported coordination has to occur in the dual tasks appears to be accomplished by the sum of the activation in the single tasks, without any overadditivity.

The constraint on the total amount of activation was manifested not only in the association areas, but also in the sensory areas, where the activation decreased very substantially from the single tasks to the dual tasks. Previous studies have also reported a decrease in activation in a sensory area when a second computational demand was placed on the same area or on another area (Rees et al., 1997; Vandenberghe et al., 1997). For example, the amount of activation in V5 associated with the perception of irrelevant moving dots was smaller during the performance of a more demanding visual word judgement task (deciding whether it had two syllables) than during a less demanding task (whether it was in uppercase) (Rees et al., 1997). The neural mechanisms underlying the word judgement tasks and the perception of motion were apparently limited by a common resource constraint, affecting the amount of activation in V5. The current results place the previous findings in a broader context. The constraint may arise not simply because one cannot look at two objects at the same time. Even when the neural systems involved are as separately located as the visual and auditory cortex, there appears to be a constraint on the amount of activation that can support them simultaneously. The limitations on the sensory area activation may well be reciprocally related to the limitations on association area activation. It may be that the limitations on the association areas impose a top-down influence on sensory areas, implementing an "attentional" effect. At the same time, the limitations on the sensory areas may constrain how much information reaches the association areas, implementing a bottom-up constraint. In a complex interactive system, constraints originating in one component may have an impact on other system components. The existence of such intersystem constraints demonstrates the nonindependence between the systems that support language and the systems that support high-level cognition, both at the sensory and at the association cortex level.

In examining the brain activation that occurs during the performance of concurrent tasks, it is useful to consider that when the two tasks make use of the very same cortical area, the resulting BOLD response is likely to be less than the sum of the responses to each task alone. The reason is that the increase in the BOLD response with the computational demand is nonlinear, suggesting that there might be a saturation of the BOLD response at high levels of demand (Friston et al., 2000). This concern would apply in the dual 
task condition in only those ROI's which were activated by both tasks when they were performed alone. For example, the prefrontal areas were activated by both tasks alone, so if underadditivity had been observed in these areas, it could have been attributed to non-linearity or saturation of the BOLD response. However, in these prefrontal areas, additivity was observed, although at a more molar level (applying to sums of voxels) than the nonlinearity is thought to apply. Perhaps the two tasks activated different voxels in any given prefrontal ROI, such that the issue of BOLD nonlinearity would not apply.

The existence of activation constraints, however their detail turns out, indicates that there is an interdependence among various parts of the cortex, determined in part by the cumulative demands that they impose on brain function in the performance of particular cognitive tasks. This perspective challenges the common assumption of neuroscience and neuropsychology that the modularity and interaction of various cortical systems is a function of only their fixed structural properties, such as anatomical locus and direct connectivity, and suggests instead that constraints apply within a dynamically configured large-scale cortical network recruited to perform the task or tasks at hand.

\section{ACKNOWLEDGMENTS}

This research was funded in part by Air F orce Office of Sponsored Research (Grant F-49620-97-1-0368), National Institute for Neurological Disorders and Stroke (Grant PO1NS35949), and National Institute of Mental Health (Grant MH29617). Correspondence and requests for materials should be addressed to Dr. Marcel J ust, Email: just+ @cmu.edu, or Department of Psychology, Carnegie MelIon University, Pittsburgh, PA 15213.

\section{REFERENCES}

Baddeley, A. D. 1986. Working Memory. Clarendon Press, Oxford, England.

Bandettini, P. A., Wong, E. C., Hinks, R. S., Tokofsky, R. S., and Hyde, J . S. 1992. Time course EPI of human brain function during task activation. Magn. Reson. Med. 25: 390-397.

Carpenter, P. A., J ust, M. A., Keller, T., Eddy, W. F., and Thulborn, K. R. 1999. Graded functional activation in the visuospatial system with the amount of task demand. J . Cogn. Neurosci. 11: 9-24.

Cohen, M. S., Kosslyn, S. M., Breiter, H. C., DiGirolamo, G. J ., Thompson, W. L., Anderson, A. K., Bookheimer, S. Y., Rosen, B. R., and Belliveau, J. W. 1996. Changes in cortical activity during mental rotation: A mapping study using functional MRI. Brain 119: $89-100$.
Corbetta, M. 1998. Frontoparietal cortical networks for directing attention and the eye to visual locations: I dentical, independent, or overlapping neural systems? Proc. Natl. Acad. Sci. USA 95: 831838.

D’Esposito, M., Detre, J . A., Alsop, D. C., Shin, R. K., Atlas, S., and Grossman, M. 1995. The neural basis of the central executive system of working memory. Nature 378: 279-281.

Eddy, W. F., Fitzgerald, M., Genovese, C. R., Mockus, A., and Noll, D. C. 1996. Functional imaging analysis software-Computational olio. A. Prat. In Proceedings in Computational Statistics, pp. 3949. Heidelberg, Physica-Verlag.

Friston, K. J ., Mechelli, A., Turner, R., and Price, C. J . 2000. Nonlinear responses in fMRI: The balloon model, Volterra kernels, and other hemodynamics. Neuroimage 12: 466- 477.

J ust, M. A., Carpenter, P. A., Keller, T. A., Eddy, W. F., and Thulborn, K. R. 1996. Brain activation modulated by sentence comprehension. Science 274: 114-116.

Klingberg, T., and Roland, P. E. 1997. Interference between two concurrent tasks is associated with activation of overlapping fields in the cortex. Cogn. Brain Res. 6: 1-8.

Lazar, N. A., Eddy, W. F., Genovese, C. R., and Welling, J . Statistical Issues in fMRI for brain imaging. Int. Statist. Rev., in press.

Mesulam, M.-M. 1990. Large-scale neurocognitive networks and distributed processing for attention, language and memory. Ann. Neurol. 28: 597-613.

Michael, E. B., Keller, T. A., Carpenter, P. A., and J ust, M. A. In press. An fMRI investigation of sentence comprehension by eye and by ear: Modality fingerprints on cognitive processes. Hum. Brain Mapp.

Norman, D. A., and Bobrow, D. G. 1975. On data-limited and resource-limited processes. Cogn. Psychol. 7: 44-64.

Rademacher, J ., Galaburda, A. M., Kennedy, D. N., Filipek, P. A., and Caviness, V. S., J r. 1992. Human cerebral cortex: Localization, parcellation, and morphometry with magnetic resonance imaging. J . Cogn. Neurosci. 4: 352-374.

Rees, G., Frith, C. D., and Lavie, N. 1997. Modulating irrelevant motion perception by varying attentional load in an unrelated task. Science 278: 1616-1619.

Schlosser, M. J ., Aoyagi, N., Fulbright, R. K., Gore, J . C., and McCarthy, G. 1998. Functional MRI studies of auditory comprehension. Hum. Brain Mapp. 6: 1-13.

Shepard, R. N., and Metzler, J. 1971. Mental rotation of three dimensional objects. Science 171: 701-703.

Tagaris, G. A., Kim, S.-G., Strupp, J . P., Andersen, P., U gurbil, K., and Georgopoulos, A. P. 1997. Mental rotation studied by functional magnetic resonance imaging at high field (4 Tesla): Performance and cortical activation. J . Cogn. Neurosci. 9: 419-432.

Vandenberghe, R., Duncan, J ., Dupont, P., Ward, R., Poline, J .-B., Bormans, G., Michiels, J., Mortelmans, L., and Orban, G. A. 1997. Attention to one or two features in left or right visual field: A positron emission tomography study. J . Neurosci. 17: 3739-3750.

Wickens, C., Kramer, A., Vanasse, L., and Donchin, E. 1983. Performance of concurrent tasks: A psychophysiological analysis of the reciprocity of information-processing resources. Science $\mathbf{2 2 1}$ : 1080-1082. 